OPEN ACCESS

Edited by:

Jeffrey P. Staab,

Mayo Clinic, United States

Reviewed by:

Vincenzo Marcelli,

Local Health Authority Naples 1

Center, Italy

Giuseppe Chiarella,

University of Catanzaro, Italy

${ }^{*}$ Correspondence:

Monica P. Mallampalli

monica@migrainecollaborative.org

tThese authors have contributed equally to this work and share first authorship

¥These authors have contributed equally to this work and share last authorship

$\$$ These authors share senior authorship

Specialty section:

This article was submitted to Neuro-Otology,

a section of the journal

Frontiers in Neurology

Received: 10 November 2021

Accepted: 29 November 2021

Published: 03 January 2022

Citation:

Mallampalli MP, Rizk HG

Kheradmand A, Beh SC, Abouzari M, Bassett AM, Buskirk J, Ceriani CEJ, Crowson MG, Djalilian H, Goebel JA,

Kuhn JJ, Luebke AE, Mandalà M, Nowaczewska M, Spare N, Teggi R, Versino $M$, Yuan $\mathrm{H}$, Zaleski-King A, Teixido M and Godley F (2022) Care Gaps and Recommendations in Vestibular Migraine: An Expert Panel Summit. Front. Neurol. 12:812678. doi: 10.3389/fneur.2021.812678

\section{Care Gaps and Recommendations in Vestibular Migraine: An Expert Panel Summit}

\author{
Monica P. Mallampalli ${ }^{1 *+}$, Habib G. Rizk ${ }^{2 \dagger}$, Amir Kheradmand ${ }^{3 \S}$, Shin C. Beh ${ }^{4 \S}$, \\ Mehdi Abouzari ${ }^{5}$, Alaina M. Bassett ${ }^{6}$, James Buskirk ${ }^{7}$, Claire E. J. Ceriani ${ }^{8}$, \\ Matthew G. Crowson ${ }^{9}$, Hamid Djalilian ${ }^{5}$, Joel A. Goebel ${ }^{10}$, Jeffery J. Kuhn ${ }^{11}$, \\ Anne E. Luebke ${ }^{12}$, Marco Mandalà ${ }^{13}$, Magdalena Nowaczewska ${ }^{14}$, Nicole Spare ${ }^{8}$, \\ Roberto Teggi ${ }^{15}$, Maurizio Versino ${ }^{16}$, Hsiangkuo Yuan ${ }^{8}$, Ashley Zaleski-King ${ }^{17}$, \\ Michael Teixido ${ }^{1 \neq \S}$ and Frederick Godley ${ }^{1 \neq \S}$
}

${ }^{1}$ Department of Research, Association of Migraine Disorders, North Kingstown, RI, United States, ${ }^{2}$ Department of Otolaryngology-Head and Neck Surgery, Medical University of South Carolina, Charleston, SC, United States, ${ }^{3}$ Department of Otolaryngology-Head and Neck Surgery, The Johns Hopkins University School of Medicine, Baltimore, MD, United States, ${ }^{4}$ Department of Neurology and Neurotherapeutics, University of Texas Southwestern Medical Center, Dallas, TX, United States, ${ }^{5}$ Department of Otolaryngology-Head and Neck Surgery, University of California, Irvine, Irvine, CA, United States, ${ }^{6}$ Department of Otolaryngology, Keck School of Medicine, University of Southern California, Los Angeles, CA, United States, ${ }^{7}$ Department of Otolaryngology, University of Miami Miller School of Medicine, Miami, FL, United States, ${ }^{8}$ Jefferson Headache Center, Thomas Jefferson University, Philadelphia, PA, United States, ${ }^{9}$ Department of Otolaryngology-Head and Neck Surgery, Mass Eye \& Ear and Harvard Medical School, Boston, MA, United States, ${ }^{10}$ Department of Otolaryngology-Head and Neck Surgery, Washington University School of Medicine, Saint Louis, MO, United States, ${ }^{11}$ Department of Research, Bayview Physicians Group, Chesapeake, VA, United States, ${ }^{12}$ Biomedical Engineering and Neuroscience, University of Rochester Medical Center, Rochester, NY, United States, ${ }^{13}$ Otolaryngology Unit, University of Siena, Siena, Italy, ${ }^{14}$ Department of Otolaryngology, Head and Neck Surgery, Laryngological Oncology, Nicolaus Copernicus University, Torun, Poland, ${ }^{15}$ Department of Otolaryngology, San Raffaele Scientific Hospital, Milan, Italy,

${ }^{16}$ Neurology and Stroke Unit, ASST Sette Laghi, Circolo Hospital, Varese, Italy, ${ }^{17}$ Department of Speech-Language Pathology \& Audiology, Towson University, Towson, MD, United States

Vestibular migraine (VM) is an increasingly recognized pathology yet remains as an underdiagnosed cause of vestibular disorders. While current diagnostic criteria are codified in the 2012 Barany Society document and included in the third edition of the international classification of headache disorders, the pathophysiology of this disorder is still elusive. The Association for Migraine Disorders hosted a multidisciplinary, international expert workshop in October 2020 and identified seven current care gaps that the scientific community needs to resolve, including a better understanding of the range of symptoms and phenotypes of $\mathrm{VM}$, the lack of a diagnostic marker, a better understanding of pathophysiologic mechanisms, as well as the lack of clear recommendations for interventions (nonpharmacologic and pharmacologic) and finally, the need for specific outcome measures that will guide clinicians as well as research into the efficacy of interventions. The expert group issued several recommendations to address those areas including establishing a global VM registry, creating an improved diagnostic algorithm using available vestibular tests as well as others that are in development, conducting appropriate trials of high quality to validate current clinically 
available treatment and fostering collaborative efforts to elucidate the pathophysiologic mechanisms underlying VM, specifically the role of the trigemino-vascular pathways.

Keywords: vestibular migraine, chronic migraine (CM), trigemino-vascular pathway, vertigo-pathophysiology, vestibular disorders

\section{INTRODUCTION}

Vestibular migraine (VM) is a migraine disorder which presents with heterogeneous symptomatology and with several overlapping phenotypes. VM remains largely underdiagnosed and knowledge of its pathophysiology is limited (1). It is typically characterized by recurrent vestibular symptoms associated with migraine symptoms (headaches of a migraine quality and/or photophobia and phonophobia and/or visual aura) and has been considered among the most common causes of dizziness (2). Risk factors associated with VM include young age (below 40 years), female sex, a history of anxiety and depression and prior head trauma (3). Previously, other terms have been used for VM which include benign recurrent vertigo, migraine-associated dizziness, migraine-associated vertigo, migrainous vertigo, and migraine-related vestibulopathy.

Epidemiological data confirm that migraine-related syndromes that include a spectrum of vestibular symptoms are the most common cause of vertigo and dizziness in adults and children (4). While VM can develop at any age, it generally affects persons with a long-established history of migraine disease (5). It is diagnosed with an average delay of 8.4 years after the first onset of migraine (6).

Since the early development of VM as a distinct diagnosis in the 1980's, the estimated prevalence of VM in the general population is not precisely known because of the general underdiagnosis of this condition (7). According to Neuhauser et al. VM accounts for about $7 \%$ of patients seen in dizziness clinics and $9 \%$ of patients seen in migraine clinics (5). Other case series report that $16-34 \%$ of patients seen for dizziness in an otolaryngology clinic have a vestibular migraine (8-10). A national telephone interview survey in Germany by Neuhauser et al. estimated the 1-year and a lifetime prevalence of VM to be $0.89 \%$ and $1 \%$ in the adult general population (11). In a more recent study by Formeister et al. within the United States (U.S.), based on a population-based survey from the 2008 National Health Interview Survey (NHIS), the prevalence of VM in adults was estimated to be $2.7 \%$ (3). This is higher than the estimated prevalence of benign paroxysmal positional vertigo (BPPV) of $1.6 \%$ and that of Meniere's disease (MD) $(0.2-0.5 \%)(12,13)$.

On October 4, 2020, the Association of Migraine Disorders (AMD) hosted a half-a-day virtual workshop to discuss and

\footnotetext{
Abbreviations: VM, vestibular migraine; MC, migraine cephalgia; BPPV, benign paroxysmal positional vertigo; MD, Meniere's Disease; MDDS, Mal de debarquement syndrome; PPPD, persistent postural perceptual dizziness; TOAEs, transient evoked otoacoustic emissions; DPOAEs, distortion product otoacoustic emissions; ABR, auditory brainstem response; VEMP, vestibular-evoked myogenic potentials; SVV, subjective visual vertical; SVH, subjective visual horizontal; TVS, trigemino-vascular system; CGRP, calcitonin gene-related peptide; SSRIs, selective serotonin reuptake inhibitors; SNRIs, serotonin and norepinephrine reuptake inhibitors; PROMs, patient reported outcomes measures.
}

identify research gaps in VM with the goal of identifying initiatives that would advance research in this field. The basis for convening this meeting was to evaluate the state of diagnosis, pathophysiology, and treatment of VM and furthermore, to answer some of the many challenging and unanswered questions related to this disease. Participants included experts from several medical specialties including otolaryngology, neurotology, neurology, audiology, and physical therapy. Based on the discussions at the workshop, this report highlights existing care gaps, supporting evidence and recommendations to address these gaps.

\section{CARE GAP 1: LACK OF UNIVERSALLY ACCEPTED RANGE OF VM PHENOTYPES AND UNCLEAR SPECTRUM OF VM DISORDERS}

In 2012, diagnostic criteria for VM were put forth by the International Headache Society (ICHD-3) and Bárány society which replaced the historical most accepted Neuheuser criteria $(14,15)$. Currently, there are multiple classification criteria for VM. The most restrictive is the ICHD-3 listed in Table 1 which acknowledges only definite VM. The Bárány society criteria makes provision for a separate category called probable vestibular migraine (Table 1) and in clinical practice, most patients who benefit from migraine therapy would fall in this latter category.

Current ICHD-3 and Bárány Society diagnostic criteria of vestibular migraine leave many endophenotypes/subtypes of VM unrecognized. For instance, dizziness per se is not exclusively a vestibular symptom qualifiable for VM diagnosis. To be considered, dizziness must be triggered by head motion and must be associated with nausea or other migraine symptoms. The current ICHD-3 symptoms that are symptoms qualifiable exclude certain dizziness phenotypes such as rocking and displacement in place seen in disorders such as Mal de debarquement (MDDS), Persistent Postural Perceptual Dizziness (PPPD), along with visual motion sensitivity. The rationale for specifying moderate or severe symptom severity is likely included to avoid duplicative diagnoses, or misattribution of dizziness symptoms (i.e., imbalance) when the primary symptom is headache. Evidence from current and future studies will help to elucidate the features of vestibular symptoms in VM and revise that point of the diagnostic criteria.

Epidemiological studies have documented the relationship between migraine headache and vertigo, suggesting an association between the two, yet the underlying neuronal pathophysiology is relatively unknown (16-18). Migraine headache typically predates the onset of vertigo with the mean duration of 8 years (6). Mean age of onset of headaches is 
TABLE 1 | Diagnostic criteria for VM as proposed by International Headache Society and Bárány Society.

\author{
Vestibular Migraine: \\ A. At least five episodes with vestibular symptoms lasting 5 min to $72 \mathrm{~h}$. \\ B. Current or previous history of migraine with or without aura. \\ C. One or more migraine features with at least $50 \%$ of the vestibular episodes: \\ b. Photophobia and phonophobia \\ c. Visual aura \\ D. Not better accounted for by another vestibular diagnosis or ICHD diagnosis \\ Probable Vestibular Migraine: \\ A. At least five episodes with vestibular symptoms lasting 5 min to $72 \mathrm{~h}$. \\ B. Only one of the criteria $\mathrm{B}$ and $\mathrm{C}$ for vestibular migraine is fulfilled. \\ C. Not better accounted for by another vestibular diagnosis or ICHD diagnosis.
}

a. Headache with at least two of: one sided location, pulsating quality, moderate or severe pain intensity, aggravation by routine physical activity

28 years old and mean age of dizziness onset is 49 years old. Patients with a synchronous or a combined presentation are younger (19). Additionally, the prevalence of vertigo is high in migraine patients and similarly, patients with dizziness often have a history of migraine (20). In the Migraine and Neck Pain Study, $30 \%$ of adult patients reported episodic vertigo anytime during the migraine attack with $16 \%$ having vertigo at the onset of headache, with $10 \%$ patients reporting episodic vertigo within $2 \mathrm{~h}$ before the headache and 3\% patients experiencing episodic vertigo as a premonitory symptom up to $48 \mathrm{~h}$ before the headache. Nausea and vomiting were found to be associated with headaches and vertigo (19). Almost a third of these patients in the Migraine and Neck Pain Study could be diagnosed with VM or at least probable VM, according to the Bárány society criteria, supporting the association between migraine headache and vertigo (20). Symptoms such as cyclic vomiting, recurrent abdominal pain and atopy are also commonly observed in migraine patients (21). Considering there is an overlap of symptoms, both migraine and vestibular migraine may be part of a disease spectrum sharing an underlying pathophysiology.

Recommendation 1: (a) Establish a global VM patient registry that maintains uniform quality data and documents the natural history of vestibular symptoms in relation to migraine disease, (b) Refine the international, evidence-based diagnostic criteria for VM to account for the various symptoms reported by VM patients.

\section{CARE GAP 2: VM IS CURRENTLY UNDERDIAGNOSED IN THE GENERAL POPULATION AND MULTIPLE ASSOCIATED CO-MORBIDITIES ARE UNDERRECOGNIZED}

Diagnosis for VM primarily relies on clinical history due to the lack of clinically available and reliable diagnostic laboratory tests. Since several epidemiological studies have supported the relationship between migraine and VM, the diagnosis of VM is very much dependent on the presence of migraine symptoms (22). Symptoms of dizziness and vertigo may be spontaneous, positional, visually induced, or head-motion induced, and are described in many ways by patients. Additionally, the timing of the attacks can also vary from minutes to days making the diagnosis of this disease very complex. For instance, as awareness of the relationship of migraine and vertigo has grown, many patients previously assigned a diagnosis of basilar migraine are now known to have VM. Basilar migraine is now better known as migraine with brainstem aura where vertigo and at least one other brainstem neurologic symptom (excluding motor weakness) must precede the headaches.

Several comorbidities are also known to be associated with VM including neurotologic conditions such as BPPV and MD. The symptoms of these conditions may overlap with those of VM symptoms and confuse the diagnosis of VM, especially when patients with other vestibular disorders may have superimposed, secondary migraine symptoms and not have $\mathrm{VM}$ as a primary cause of their symptoms (22). Psychiatric comorbidities, including anxiety, somatoform dizziness/chronic subjective dizziness may coexist with VM as well $(23,24)$. Furthermore, acknowledging that patients may have more than one vestibular diagnosis contributing to their symptoms is essential to providing accurate treatment and conducting accurate research (25).

There are suggestions that migraine mechanisms can affect the inner ear, that BPPV and MD can be complications of a migraine process, and that these conditions are potentially in pathophysiologic continuum with VM in which some patients manifest pure central disease, others manifest pure peripheral disease, and others have manifestations of both $(26,27)$. Patients with VM may exhibit tinnitus, aural pressure, and fluctuations in hearing. Hearing loss however does not tend to progress as it does in MD (28). Early on, MD patients may exhibit isolated episodic vertigo without otologic symptoms. Despite having a formal diagnostic criterion for VM and $\mathrm{MD}$, it is becoming clearer that the diagnostic criteria for VM need to be refined and broadened (29). For instance, Frejo et al. define five endophenotypes for MD: idiopathic or classic, posttraumatic/delayed, autoimmune, familial or associated with migraine (30). The current ICHD3 criteria acknowledge that there are patients with features of both disorders (with cochleovestibular symptoms of ear 
fullness/pressure, periodic or fluctuating tinnitus, fluctuating hearing loss in the setting of vestibular migraine or of a migraine disorder) and recommend treating the patients for MD especially if there is compelling audiometric confirmation. In addition to understanding the multiple phenotypic presentations of VM, patients with classifiable peripheral disease such as MD and recurrent BPPV responding to migraine management and with migraine features during attacks should potentially be considered to be on the same spectrum $(31,32)$. This broadening of recognized symptoms should be expanded to include patients with chronic rocking dizziness and patients with disorientation in space, as these symptoms are commonly reported by migraine patients. While MDDS is currently considered a separate entity, it is the hallmark condition causing rocking dizziness. Multiple studies show high prevalence of migraines especially in "spontaneous mal de debarquement" as well as high prevalence of new-onset headaches in patients with new onset MDDS $(8,33,34)$.

Recommendations 2: (a) Enhance provider education, especially among practitioners who manage patients with dizziness, around VM and its potential subtypes including complexity of symptoms that may occur on a continuum, (b) Consider refining ICD coding for VM and possible subtypes as has been done for migraine headache.

\section{CARE GAP 3: LACK OF A KNOWN DIAGNOSTIC LABORATORY TESTING OR AN OBJECTIVE MARKER AVAILABLE FOR CLINICIANS}

The current diagnostic criteria of VM are only clinical and based on the identification of the features outlined in the consensus of the International Headache and Bárány Societies in the ICHD-3 $(14,15)$. There is no pathognomonic clinical sign for vestibular migraine and there are no gold standard diagnostic tests for VM.

However, certain paraclinical tests are administered in the clinical setting. Auditory and vestibular testing has some value in the comprehensive evaluation of these patients. Classic audiometry can put in evidence a low frequency hearing loss suggestive of MD or concomitant MD. Other audiometric tests are not widely used in clinical practice. However, transient evoked otoacoustic emissions (TOAEs), distortion product otoacoustic emissions (DPOAEs), and auditory brainstem response $(\mathrm{ABR})$ at high and low repetition rate frequencies show nonspecific abnormalities in nearly two thirds of patients with chronic migraine (35).

In addition, while vestibular testing cannot identify the presence of VM, it can put in evidence a concurrent dysfunction such as uncompensated vestibular hypofunction (due to peripheral or central mechanisms), comorbid BPPV as well as utricular/saccular dysfunction (35). Additionally, certain patterns can emerge on testing as well and help guide the diagnosis and/or the treatment plan (36). For instance, motion sensitive individuals tend to have longer time decay constants on step velocity rotary chair testing $(37,38)$. Some patients with vestibular migraines have caloric response asymmetry or may have oculomotor deficits or positional nystagmus with central features (39-41). While some studies points toward peripheral dysfunction in the setting of vestibular migraine, one should bear in mind that a caloric asymmetry can still indicate a separate vestibular disorder or a preexisting vestibular dysfunction which in itself may be a cause of migraines. Other observed patterns include the fact that VM patients are less likely to complete a full battery of vestibular tests and are more likely to develop an attack following testing $(42,43)$.

Vestibular-evoked Myogenic Potentials (VEMPs), a measure of saccular and utricular function, can separate in some instances VM from MD but the data is not clear-cut and there are no consistently reliable abnormalities (44-46). A recent systematic review did not support the role of cervical VEMP alone to aid in diagnosing VM (47). Rizk et al. (48) looked at the negative predictive value of the presence of an oVEMP and/or cVEMP response in patients presenting with a differential diagnosis of $\mathrm{VM}$ vs MD and showed that a present response in this specific clinical situation has a 93\% negative predictive value. Subsequent studies suggested using VEMPs as part of a diagnostic algorithm.

On the other hand, current research is looking into other tools that could identify a patient with VM and differentiate them from other pathologies, namely MD. Tests of perceptual threshold, which are still not clinically available, show reduced dynamic tilt thresholds in vestibular migraine patients (49). The measurement of subjective visual vertical (SVV) and subjective visual horizontal $(\mathrm{SVH})$ can serve as diagnostic indicators of dysfunction in spatial orientation (50). Abnormal SVV and SVH measurements occur in a significant number of individuals with VM, especially during head tilt where the brain is challenged to maintain spatial orientation $(51,52)$. The larger SVV errors in VM compared with healthy controls suggest abnormal sensory integration for spatial orientation in these patients (51). Thus, incorporating head-roll tilt during static and dynamic SVV conditions rather than in the traditional head upright position can produce more distinctive findings in migraine patients.

The functional head impulse test with and without optokinetic stimulation shows promise in identifying VM patients since it highlights dynamic visual dependence which is a hallmark manifestation of VM (53). The usefulness of this test in make a differential diagnosis with MD is to be evaluated (54).

Finally, there has been a quest to identify a readily accessible biomarker for VM. A recent study has identified a differential proinflammatory signature separately for patients with VM and MD where certain cytokines and chemokines were elevated. These preliminary studies suggest VM can be differentially diagnosed from healthy individuals and patients with MD using a small cytokine panel retrieved from a blood sample (IL-1 $\beta$, IFN $\gamma$, CCL3, CCL22, and CXCL1) thereby improving the clinical management of VM (55).

Recommendation 3: (a) Create an improved diagnostic algorithm for VM using available vestibular function testing and consider incorporating perceptual threshold testing into clinical practice, (b) Pursue further research to identify a circulating biomarker to differentiate VM from MD. 


\section{CARE GAP 4: PATHOPHYSIOLOGIC MECHANISMS EXPLAINING THE VARIOUS PHENOTYPES AND FORMS OF VM (EPISODIC VS. CHRONIC) AS WELL AS ASSOCIATED SYMPTOMS (SPATIAL DISORIENTATION, HEARING LOSS, VERTIGO) ARE STILL UNCLEAR}

In general, very little is known about the pathophysiology of VM. Given the multiple potential interactions between the trigeminal and vestibular systems, it is likely that VM symptoms have a multifactorial etiopathophysiologic basis. Several possible mechanisms have been proposed based on the general understanding of migraine pathophysiology. Potential involvement and interactions between the trigemino-vascular system (TVS), nociceptive brain stem centers, thalamocortical network, and vestibular system have also been suggested to explain the underlying pathogenesis linking various VM symptoms (1).

Headaches are thought to result from an increased activity or sensitization within the TVS and nociceptive brainstem and thalamocortical centers. Animal models of chronic migraine suggest that migraine-mediated sensitization of the trigeminal nuclei, through its neural projections to vestibular nuclei, might affect the sensitivity of vestibular nuclei resulting in migraineassociated vestibular dysfunction (56). Certain neuropeptides such as calcitonin gene-related peptide (CGRP) and substance $\mathrm{P}$ cause neuroinflammation leading to pain or allodynia (central sensitization) in migraine patients. Some of these same neuropeptides (such as CGRP) are also expressed in the vestibular system and could be involved in VM pathophysiology (56). In fact, systemic CGRP injection has been shown to cause light-aversion (photosensitivity) in mice which can be blocked by CGRP antagonists or monoclonal antibodies (57). Furthermore, using a rat model of chronic migraine, Zhang et al. (56) demonstrated the possibility of sensitization of vestibular nucleus neurons to impair vestibular function after chronic migraine and were able to restore vestibular dysfunction in following anti-CGRP treatment (56). An increase in CGRP levels in both sensitized trigeminal and vestibular nuclei suggest that CGRP may have a critical role in the transformation to chronic sensitization. Therefore, just as CGRP enhances the abnormal pain sensitivity, it is possible that it might also increase the sensitivity of the balance system and can explain the spontaneous or sustained nature of VM (56).

Trigeminal innervation of the labyrinth vessels links the nociceptive trigeminal system to the vestibular system. Connections between brainstem vestibular nuclei and the structures that modulate trigeminal nociceptive inputs throughout the brainstem may play some part in VM. Neuroimaging techniques suggest an altered modulation in the integration and processing of vestibular and nociceptive information with the network from the vestibular sensors through the brainstem to the cortex. In this context, genetic variables can affect the excitatory-inhibitory balance involved in neural processing of sensory information, vestibular inputs, and pain (56).

Functional imaging data suggests the involvement of the multisensory vestibular thalamocortical networking in the pathophysiology of VM $(1,58,59)$. Dizziness and spatial disorientation are thought to be caused by abnormal sensory modulation or integration within the thalamocortical network (1). Several findings indicate central dysfunction related to vestibular processing in patients such as perceptual dysfunction, abnormal spatial orientation during head tilt, elevated vestibuloperceptual threshold after adaptation to visual motion $(51,60)$. These findings suggest that in general the perceptual functions of vestibular system are on a spectrum with VM on one end with low or distorted perception threshold and high level of symptoms (1). While, on the other end of the spectrum, there are those with high perceptual thresholds (such as ballet dancers) and low symptoms who show shorter durations of vestibular ocular response and perception of rotation (time constant) with whole body rotation $(1,61)$. Thus, an effective treatment strategy can be envisioned that could move patients toward the middle of the spectrum by increasing motion perception thresholds. Therefore, gaining insights into central processes that result in dizziness, vertigo, and spatial disorientation is an important step toward devising effective treatment strategies (1).

Altered activity of the vestibular system is thought to cause vestibular ocular dysfunction or vestibular hypersensitivity associated with migraine features (1). Also, a recent crosssectional study suggested that cortical interactions between the visuo-vestibular system are abnormal in vestibular migraine patients (1). Results from peripheral vestibular testing in VM patients has shown inconsistent results indicating vestibular dysfunction and some of this dysfunction could be related to the modulation of inner ear function by migraine-related mechanisms. Furthermore, the sensory neuroepithelia within the vestibular sensory pathways are thought to be influenced by a diverse number of neuroactive substances that may act to enhance or inhibit the effect of the primary neurotransmitters such as glutamate and acetylcholine (62).

In addition, it is also hypothesized that integration of canal and otolith inputs for motion perception could be abnormal in VM, a function that is mediated by the cerebellar nodulus and uvula and/or possibly the thalamus (63). It was further hypothesized that elevated levels of CGRP during and between vertigo episodes could reduce Purkinje cell activity, thereby disinhibiting the neurons in the vestibular nuclei that receive their projections and contribute to the velocity storage network (64). Velocity storage has been linked to the synthesis of canal and otolith cues and motion sickness and aberrant control of this network could contribute to the vertigo and motion intolerance in $\operatorname{VM}(49,63)$.

Finally, the vascular circulation of the inner ear receives innervation from the trigeminal nerve through the basilar artery and the anterior inferior cerebellar artery. Chemical and electrical stimulations of the trigeminal nerve may cause a significant increase in inner ear blood flow and neuroinflammation - changes in intravascular permeability and plasma protein 
extravasation into the inner ear (65). Vasospasm of the cochlear and/or vestibular branches of the internal auditory artery might explain sudden episodes of hearing loss and/or vertigo associated with migraine (49).

Recommendation 4: (a) Promote collaborative research (between laboratory scientists and clinicians) to study central and peripheral mechanisms of VM symptoms (b) Identify the effect of modulating the TVS, possibly by blocking CGRP, in the inner ear specifically and then understanding its effects on neuroepithelium in the auditory and vestibular system to provide some clues to VM pathophysiology.

\section{CARE GAP 5: LACK OF UNDERSTANDING OF THE BIOLOGICAL DIFFERENCES UNDERLYING GENDER DISPARITY IN VM}

While VM can occur at any age and between both sexes, it peaks in middle age and is 2-3 times more prevalent in women than in men $(6,66,67)$. A common pattern/typical patient seen in a clinic is a woman with a history of classic or common migraines with improved symptoms following menopause but presenting with new-onset vestibular symptoms years after headaches have diminished $(1,68)$.

Changes in hormonal levels can be associated with episodes of vestibular migraine which can sometimes be observed in the perimenstrual period (69-71). Vestibular symptoms in women can also become more pronounced around the time of perimenopause. Dizziness is a common symptom in the late stage of menopausal transition and an unstable hormonal state is considered to be its trigger. While the role of sex hormones (estrogen and progesterone) in VM is not yet understood they are thought to influence various neurotransmitter systems in the brain that are associated with migraine activation such as serotonergic, glutaminergic, GABAergic, noradrenergic, and opioid systems (72).

Recommendation 5: Promote studies to understand possible associations between hormonal changes and vestibular symptoms across the lifespan.

\section{CARE GAP 6: LACK OF APPROPRIATE COMBINATION AF PHARMACOLOGIC AND NONPHARMACOLOGIC MEASURES TO TREAT VESTIBULAR MIGRAINE AS WELL AS OF AN APPROPRIATE STEPWISE MANAGEMENT ALGORITHM}

The current treatment approach of the VM patient is not well codified and is comprised of nonpharmacologic measures as well as medications prescribed (for the most part) in an off-label indication, and rehabilitative measures of uncertain potential. Most measures are based on empiric evidence or extrapolated from the treatment of typical migraine headaches.

Non-pharmacological measures such as diet, sleep hygiene and avoidance of triggers are recommended the same way they are for migraine headaches. Greater occipital nerve blocks and vagal nerve neuromodulators can be helpful as well $(72,73)$. Nutraceuticals have been proven to be effective in prevention of migraine headaches have not yet been studied thoroughly studied for VM.

The mainstay of the management of vestibular migraine is prophylactic medication when VM attacks are frequent and severe. Migraine headache prophylactic medications have been traditionally used to treat VM and observational studies with these medications have shown benefits (74). Pharmacological drugs for VM have been used for acute treatment or as preventative treatments and are classified into two groups based on their mechanism of action. One class of drugs act on the neurotransmitters and their receptors. The second class of drugs act on voltage-gated channels (75). The details of the current drugs used for treatment of VM along with their daily dosage and common side effects have been reviewed extensively before $(74,76,77)$. Typically, the choice of medication for treating VM is guided by its side effect profile and the comorbidities of patients. Interestingly, these treatment recommendations have been based on observational studies, physicians' experiences, familiarity with the medications, patient comorbidities and side effects of various interventions $(74,78)$.

Treatment trials for $\mathrm{VM}$ are rare and only now starting to emerge. Previously, only two randomized controlled trials were conducted with triptans (rizatriptan and zolmitriptan) specifically for acute attacks to VM and these provided limited evidence for treating VM (79). A recent systematic review and meta-analysis of 13 studies assessed the efficacy of various therapies for prevention of VM. Treatment options analyzed included antiepileptic drugs, calcium channel blockers, tricyclic antidepressants, beta blockers, selective serotonin reuptake inhibitors (SSRIs), serotonin and norepinephrine reuptake inhibitors (SNRIs) and vestibular rehabilitation (78). All these treatments showed improvement in measured outcomes, but an established preferred treatment could not be determined due to the heterogeneity of the VM symptoms and the lack of standardized reporting on outcomes $(8,78,80)$.

Experiencing vertigo can produce anxiety, therefore treating or managing comorbidities, particularly mood disorders, can help with vestibular symptoms (81). Tricyclic antidepressants such as amitriptyline or nortriptyline, or SSRIs and benzodiazepines such as clonazepam have been recommended (82). SNRI have also been suggested as clinically safe and effective for migraine as well as VM prophylaxis but were considered inferior in response to other drugs (83).

CGRP monoclonal antibodies are novel therapies that have been approved for either prevention or treatment of migraine. Clinically we can assume that by controlling migraines in patients with CGRP monoclonal antibodies, we may be able to reduce or eliminate symptoms of VM. There is currently an active single center pilot study with randomized double-blinded placebo-controlled trial comparing the efficacy and safety of galcanezumab to placebo in the treatment of vestibular migraine (https://clinicaltrials.gov/ct2/show/ NCT04417361). Preliminary data from another monoclonal antibody eptinezumab (NCT04152083) for prevention of chronic migraine were positive, but it is yet to be approved by the FDA. 
TABLE 2 | List of care gaps and recommendations for vestibular migraine proposed by the expert panel.

\section{Care Gaps}

1. Lack of universally accepted range of VM phenotypes and unclear spectrum of VM disorders.

2. VM is currently underdiagnosed in the general population and multiple associated co-morbidities are underrecognized.

3. Lack of a known diagnostic laboratory testing or an objective marker available for clinicians.

4. Pathophysiologic mechanisms explaining the various phenotypes and forms of VM (episodic versus chronic) as well as associated symptoms (spatial disorientation, hearing loss, vertigo) are still unclear.

5. Lack of understanding of the biological differences underlying gender disparity in VM.

6. Lack of appropriate combination of pharmacologic and nonpharmacologic measures to treat vestibular migraine as well as of an appropriate stepwise management algorithm.

7. Need for better Patient Reported Outcome Measure (PROM) to quantify the impact of VM or any intervention on the patient's quality of life.

\section{Recommendations}

(1a) Establish a global VM patient registry that maintains uniform quality data and documents the natural history of vestibular symptoms in relation to migraine disease.

(1b) Refine the international, evidence-based diagnostic criteria for VM to account for the various symptoms reported by VM patients.

(2a) Enhance provider education, especially among practitioners who manage patients with dizziness, around VM and its potential subtypes including complexity of symptoms that may occur on a continuum (2b) Consider refining ICD coding for VM and possible subtypes as has been done for migraine headache.

(3a) Create an improved diagnostic algorithm for VM using available vestibular function testing and consider incorporating perceptual threshold testing into clinical practice.

(3b) Pursue further research to identify a circulating biomarker to differentiate VM from MD.

(4a) Promote collaborative research (between laboratory scientists and clinicians) to study central and peripheral mechanisms of VM symptoms.

(4b) Identify the effect of modulating the TVS, possibly by blocking CGRP, in the inner ear specifically and then understanding its effects on neuroepithelium in the auditory and vestibular system to provide some clues to VM pathophysiology.

5. Promote studies to understand possible associations between hormonal changes and vestibular symptoms across the lifespan.

(6a) Promote novel and evidence-based approaches for VM treatment.

(6b) Develop and publish guidelines on conducting and reporting randomized controlled trials of acute and preventative treatment of VM modeled on available guidelines developed by the ICHD for migraine cephalgia trials.

(6c) Conduct randomized control trials to study pharmaceutical and non-pharmaceutical approaches for VM treatment.

7. Create and validate a VM disease-specific PROM instrument using psychometrically valid methods
Serotonin receptor $5-\mathrm{HT}_{\mathrm{IF}}$ is believed to regulate the release of CGRP from vestibular nuclei (84). In 2019, FDA approved 5$\mathrm{HT}_{\mathrm{IF}}$ receptor agonist lasmiditan for treatment of acute migraine (85). Considering Lasmiditan was effective in treating headache and photophobia, it may have the potential to serve as a prophylactic treatment for VM.

Vestibular rehabilitation and natural activities that can enhance spatial perception and body coordination, such as ping-pong and dancing can be helpful to alleviate symptoms in VM patients. Vestibular rehabilitation can be useful when there is associated vestibular dysfunction or comorbid BPPV but also when patients develop maladaptive strategies that lead to visual dependence or loss of confidence in balance (82). More studies are warranted to truly understand the benefits of vestibular rehabilitation and other rehabilitative strategies in the VM patients (86). Rehabilitation should be conducted by an experienced therapist who will be able to slowly and incrementally increase the dose of the exercises to avoid causing significant symptoms since these patients are generally very motion sensitive.

Finally, current treatment strategies are focused on reducing the burden of vestibular and headache symptom burden. Often overlooked symptoms such as "brain fog," "foggy headed," "head in a cloud" and "difficulty thinking clearly" correlate with worse scores on cognitive scales and are best classified as forms of cognitive dysfunction. Cognitive dysfunction may be a cause of ongoing disability in VM patients. There is currently no clear intervention that would help address this specific symptomatology although cognitive therapy or other rehabilitative measures could be explored $(48,87)$.

Recommendation: (a) Promote novel and evidence-based approaches for VM treatment, (b) Develop and publish guidelines on conducting and reporting randomized controlled trials of acute and preventative treatment of VM modeled on available guidelines developed by the ICHD for migraine cephalgia trials (88), (c) Conduct randomized control trials to study pharmaceutical and non-pharmaceutical approaches for VM treatment.

\section{CARE GAP 7: NEED FOR BETTER PATIENT REPORTED OUTCOME MEASURE (PROM) TO QUANTIFY THE IMPACT OF VM OR ANY INTERVENTION ON THE PATIENT'S QUALITY OF LIFE}

Vestibular disorders are a major cause of absenteeism and loss of productivity at work (89). In addition, vestibular disorders in general and VM in particular have been associated with sleep disturbances, psychiatric comorbidities and cognitive dysfunction. Furthermore, chronic migraine, by itself, is considered the second most prevalent cause of disability worldwide (90). Given the prevalence of these disorders and their impact on patients, it can be inferred that vestibular disorders, particularly VM can have a major impact on a patients' quality of life. 
The current available tools such as the dizziness handicap inventory, and PROMs are not disease specific and do not capture the extent of the condition's impact (91-93). There is also a need for better PROMs for research study design (critical to improving success in NIH funded projects). Efforts are being made in that area with Sharon et al. recently describing a potential new instrument VM-PATHI (94). In addition to being able to gauge the severity of the condition, in the absence of an objective marker for diagnosis, we currently also rely on patients' reports to gauge the response to any intervention. PROMs are used in most clinical trials as outcome measures. To this end, there is a need to properly define appropriate outcome measures before designing and studying treatment interventions.

Recommendation 7: Create and validate a VM disease-specific PROM instrument using psychometrically valid methods.

\section{CONCLUDING STATEMENT}

We have identified seven broad care gaps in our understanding and management of vestibular migraine. There is a lack of definition of endophenotypes of VM and underdiagnosis of subjects with atypical symptoms or who do not fit the ICHD-3 criteria strictly. The absence of an objective diagnostic marker contributes to those gaps as well as the insufficient knowledge of pathogenesis including hormonal influence. Furthermore, there is insufficient evidence of best treatment algorithms and treatment is often initiated based on comorbidities and side effect profile without clear evidence for a stepwise approach. Finally, there is a need to develop an instrument that can capture all the dimensions of the quality of life affected by the VM condition; this will help gauge the severity of the condition but

\section{REFERENCES}

1. Huang TC, Wang SJ, Kheradmand A. Vestibular migraine: an update on current understanding and future directions. Cephalalgia. (2020) 40:107-21. doi: $10.1177 / 0333102419869317$

2. Lin HW, Bhattacharyya N. Impact of dizziness and obesity on the prevalence of falls and fall-related injuries. Laryngoscope. (2014) 124:2797801. doi: 10.1002/lary.24806

3. Formeister EJ, Rizk HG, Kohn MA, Sharon JD. The epidemiology of vestibular migraine: a population-based survey study. Otol Neurotol. (2018) 39:1037-44. doi: 10.1097/MAO.0000000000001900

4. Dieterich M, Obermann M, Celebisoy N. Vestibular migraine: the most frequent entity of episodic vertigo. J Neurol. (2016) 263 (Suppl 1):S82-9. doi: 10.1007/s00415-015-7905-2

5. Neuhauser H, Leopold M, von Brevern M, Arnold G, Lempert T. The interrelations of migraine, vertigo, and migrainous vertigo. Neurology. (2001) 56:436-41. doi: 10.1212/WNL.56.4.436

6. Thakar A, Anjaneyulu C, Deka RC. Vertigo syndromes and mechanisms in migraine. J Laryngol Otol. (2001) 115:782-7. doi: 10.1258/0022215011909251

7. Geser R, Straumann D. Referral and final diagnoses of patients assessed in an academic vertigo center. Front Neurol. (2012) 3:169. doi: 10.3389/fneur.2012.00169

8. Van Ombergen A, Van Rompaey V, Van de Heyning P, Wuyts F. Vestibular migraine in an otolaryngology clinic: prevalence, associated symptoms, and prophylactic medication effectiveness. Otol Neurotol. (2015) 36:133-8. doi: 10.1097/MAO.0000000000000596 also may be used as an outcome measure of interventions. The recommendations proposed by this group to address the care gaps, listed together in Table 2, broadly fall into three categories, namely establishing a VM registry, advancing clinical research on VM and providing education. Establishing a registry can allow for creating a treatment algorithm, a diagnostic algorithm as well as retrieval of high-quality data provided an ICD code is created. Advancing clinical research is critical as many knowledge gaps remain to this day. Encouraging and fostering collaborations with basic researchers can promote an understanding of the underlying pathophysiological mechanisms of VM and its connection to chronic migraine and other comorbidities. Although treatments are available, there is a critical need for evidence-based approaches to treating and managing VM. More clinical trials for novel treatments are needed with appropriate outcome measures along with comparative efficacy studies for existing treatments. Finally, broader education and awareness on VM, its endophenotypes and its relation to comorbidities is clearly needed for clinicians who treat patients for dizziness.

\section{AUTHOR CONTRIBUTIONS}

$\mathrm{AK}, \mathrm{SB}, \mathrm{MT}$, and FG provided critical review and input on the initial drafts. All authors contributed to manuscript revision, read, and approved the submitted version.

\section{FUNDING}

The funds used for publication fees were received from the Association of Migraine Disorders (AMD). AMD is a non-profit organization and strives to expand the understanding of migraine by supporting research, education, and awareness.
9. Huppert D, Brandt T. Dizziness and vertigo syndromes viewed with a historical eye. J Neurol. (2018) 265:127-33. doi: 10.1007/s00415-0188807-x

10. Muelleman T, Shew M, Subbarayan R, Shum A, Sykes K, Staecker H, et al. Epidemiology of dizzy patient population in a neurotology clinic and predictors of peripheral etiology. Otol Neurotol. (2017) 38:870-5. doi: 10.1097/MAO.0000000000001429

11. Neuhauser HK, Radtke A, von Brevern M, Feldmann M, Lezius F, Ziese T, et al. Migrainous vertigo: prevalence and impact on quality of life. Neurology. (2006) 67:1028-33. doi: 10.1212/01.wnl.0000237539.09942.06

12. von Brevern M, Radtke A, Lezius F, Feldmann M, Ziese T, Lempert $\mathrm{T}$, et al. Epidemiology of benign paroxysmal positional vertigo: a population based study. J Neurol Neurosurg Psychiatry. (2007) 78:710-5. doi: 10.1136/jnnp.2006.100420

13. Kim MH, Cheon C. Epidemiology and seasonal variation of Meniere's disease: data from a population-based study. Audiol Neurootol. (2020) 25:224-30. doi: 10.1159/000506921

14. Lempert T, Olesen J, Furman J, Waterston J, Seemungal B, Carey J, et al. Vestibular migraine: diagnostic criteria. J Vestib Res. (2012) 22:167-72. doi: 10.3233/VES-2012-0453

15. Hilton DB, Shermetaro C. Migraine-Associated Vertigo. Treasure Island (FL): StatPearls (2021).

16. Eggers SD, Staab JP, Neff BA, Goulson AM, Carlson ML, Shepard NT. Investigation of the coherence of definite and probable vestibular migraine as distinct clinical entities. Otol Neurotol. (2011) 32:1144-51. doi: 10.1097/MAO.0b013e31822a1c67 
17. Kayan A, Hood JD. Neuro-otological manifestations of migraine. Brain. (1984) 107:1123-42. doi: 10.1093/brain/107.4.1123

18. Baloh RW. Neurotology of migraine. Headache. (1997) 37:615-21. doi: 10.1046/j.1526-4610.1997.3710615.x

19. Teggi R, Colombo B, Albera R, Asprella Libonati G, Balzanelli C, Batuecas Caletrio A, et al. Clinical features of headache in patients with diagnosis of definite vestibular migraine: the VM-phenotypes projects. Front Neurol. (2018) 9:395. doi: 10.3389/fneur.2018.00395

20. Lampl C, Rapoport A, Levin M, Brautigam E. Migraine and episodic vertigo: a cohort survey study of their relationship. J Headache Pain. (2019) 20:33. doi: 10.1186/s10194-019-0991-2

21. Akdal G, Ozge A, Ergor G. The prevalence of vestibular symptoms in migraine or tension-type headache. J Vestib Res. (2013) 23:101-6. doi: 10.3233/VES-130477

22. Eggers SD, Neff BA, Shepard NT, Staab JP. Comorbidities in vestibular migraine. J Vestib Res. (2014) 24:387-95. doi: 10.3233/VES-140525

23. Lahmann C, Henningsen P, Brandt T, Strupp M, Jahn K, Dieterich M, et al. Psychiatric comorbidity and psychosocial impairment among patients with vertigo and dizziness. J Neurol Neurosurg Psychiatry. (2015) 86:302-8. doi: 10.1136/jnnp-2014-307601

24. Best C, Eckhardt-Henn A, Tschan R, Dieterich M. Psychiatric morbidity and comorbidity in different vestibular vertigo syndromes. Results of a prospective longitudinal study over one year. J Neurol. (2009) 256:58-65. doi: 10.1007/s00415-009-0038-8

25. Roberts RA, Jacobson GP, Hatton K. Multiple co-occurring vestibular disorders identified using the dizziness symptom profile. Am J Audiol. (2020) 29:410-8. doi: 10.1044/2020_AJA-19-00119

26. Bruss D, Abouzari M, Sarna B, Goshtasbi K, Lee A, Birkenbeuel $\mathrm{J}$, et al. Migraine features in patients with recurrent benign paroxysmal positional vertigo. Otol Neurotol. (2021) 42:461-5. doi: 10.1097/MAO.0000000000002976

27. Swaminathan A, Smith JH. Migraine and vertigo. Curr Neurol Neurosci Rep. (2015) 15:515. doi: 10.1007/s11910-014-0515-Z

28. Radtke A, Neuhauser H, von Brevern M, Hottenrott T, Lempert T. Vestibular migraine-validity of clinical diagnostic criteria. Cephalalgia. (2011) 31:90613. doi: 10.1177/0333102411405228

29. Lopez-Escamez JA, Dlugaiczyk J, Jacobs J, Lempert T, Teggi R, von Brevern $\mathrm{M}$, et al. Accompanying symptoms overlap during attacks in Meniere's disease and vestibular migraine. Front Neurol. (2014) 5:265. doi: 10.3389/fneur.2014.00265

30. Frejo L, Martin-Sanz E, Teggi R, Trinidad G, Soto-Varela A, Santos-Perez $\mathrm{S}$, et al. Extended phenotype and clinical subgroups in unilateral Meniere disease: a cross-sectional study with cluster analysis. Clin Otolaryngol. (2017) 42:1172-80. doi: 10.1111/coa.12844

31. Basura GJ, Adams ME, Monfared A, Schwartz SR, Antonelli PJ, Burkard R, et al. Clinical practice guideline: Meniere's disease. Otolaryngol Head Neck Surg. (2020) 162 (2 Suppl):S1-55. doi: 10.1177/0194599820909438

32. Bhattacharyya N, Gubbels SP, Schwartz SR, Edlow JA, El-Kashlan H, Fife T, et al. Clinical practice guideline: benign paroxysmal positional vertigo (Update). Otolaryngol Head Neck Surg. (2017) 156 (3 Suppl):S1-47. doi: $10.1177 / 0194599816689667$

33. Toda N, Shimamoto K. The influence of sympathetic stimulation on transmembrane potentials in the S-A node. J Pharmacol Exp Ther. (1968) 159:298-305.

34. Cha YH, Cui Y. Rocking dizziness and headache: a two-way street. Cephalalgia. (2013) 33:1160-9. doi: 10.1177/0333102413487999

35. Hamed SA, Youssef AH, Elattar AM. Assessment of cochlear and auditory pathways in patients with migraine. Am J Otolaryngol. (2012) 33:385-94. doi: 10.1016/j.amjoto.2011.10.008

36. Liu YF, Dornhoffer JR, Donaldson L, Rizk HG. Impact of caloric test asymmetry on response to treatment in vestibular migraine. J Laryngol Otol. (2021) 135:320-6. doi: 10.1017/S0022215121000712

37. Chua KW, Kek TL. A preliminary study: central vestibular sensitivity affects motion sickness susceptibility through the efficacy of the velocity storage mechanism. Audiol Res. (2020) 10:21-30. doi: 10.4081/audiores.2020.245

38. Hoffer ME, Gottshall K, Kopke RD, Weisskopf P, Moore R, Allen KA, et al. Vestibular testing abnormalities in individuals with motion sickness. Otol Neurotol. (2003) 24:633-6. doi: 10.1097/00129492-200307000-00017
39. Young AS, Nham B, Bradshaw AP, Calic Z, Pogson JM, D'Souza M, et al. Clinical, oculographic, and vestibular test characteristics of vestibular migraine. Cephalalgia. (2021) 41:1039-52. doi: 10.1177/03331024211006042

40. Beh SC, Masrour S, Smith SV, Friedman DI. The spectrum of vestibular migraine: clinical features, triggers, and examination findings. Headache. (2019) 59:727-40. doi: 10.1111/head.13484

41. Dieterich M, Brandt T. Episodic vertigo related to migraine (90 cases): vestibular migraine? J Neurol. (1999) 246:883-92. doi: 10.1007/s004150050478

42. Foster CA, Pollard CK. Comparison of caloric reactivity between migraineurs and non-migraineurs. J Laryngol Otol. (2015) 129:960-3. doi: 10.1017/S0022215115002066

43. Seemungal B, Rudge P, Davies R, Gresty M, Bronstein A. Three patients with migraine following caloric-induced vestibular stimulation. J Neurol. (2006) 253:1000-1. doi: 10.1007/s00415-006-0144-9

44. Taylor RL, Zagami AS, Gibson WP, Black DA, Watson SR, Halmagyi MG, et al. Vestibular evoked myogenic potentials to sound and vibration: characteristics in vestibular migraine that enable separation from Meniere's disease. Cephalalgia. (2012) 32:213-25. doi: 10.1177/0333102411434166

45. Zaleski A, Bogle J, Starling A, Zapala DA, Davis L, Wester M, et al. Vestibular evoked myogenic potentials in patients with vestibular migraine. Otol Neurotol. (2015) 36:295-302. doi: 10.1097/MAO.0000000000000665

46. Boldingh MI, Ljostad U, Mygland A, Monstad P. Vestibular sensitivity in vestibular migraine: VEMPs and motion sickness susceptibility. Cephalalgia. (2011) 31:1211-9. doi: 10.1177/0333102411409074

47. Fife TD, Colebatch JG, Kerber KA, Brantberg K, Strupp M, Lee H, et al. Practice guideline: cervical and ocular vestibular evoked myogenic potential testing: report of the guideline development, dissemination, and implementation subcommittee of the American Academy of Neurology. Neurology. (2017) 89:2288-96. doi: 10.1212/WNL.0000000000004690

48. Rizk HG, Liu YF, Strange CC, Van Ausdal CH, English RC, McRackan TR, et al. Predictive value of vestibular evoked myogenic potentials in the diagnosis of Meniere's disease and vestibular migraine. Otol Neurotol. (2020) 41:828-35. doi: 10.1097/MAO.0000000000002636

49. Lewis RF, Priesol AJ, Nicoucar K, Lim K, Merfeld DM. Dynamic tilt thresholds are reduced in vestibular migraine. J Vestib Res. (2011) 21:323-30. doi: 10.3233/VES-2011-0422

50. Chan TLH, Hale TD, Steenerson KK. Vestibular lab testing: interpreting the results in the headache patient with dizziness. Curr Neurol Neurosci Rep. (2020) 20:16. doi: 10.1007/s11910-020-01036-4

51. Winnick A, Sadeghpour S, Otero-Millan J, Chang TP, Kheradmand A. Errors of upright perception in patients with vestibular migraine. Front Neurol. (2018) 9:892. doi: 10.3389/fneur.2018.00892

52. Obrero-Gaitan E, Manrique-Navarro M, Lerida-Ortega MA, RodriguezAlmagro D, Osuna-Perez MC, Lomas-Vega R. Misperception of visual verticality in patients with primary headache disorders: a systematic review with meta-analysis. Brain Sci. (2020) 10:664. doi: 10.3390/brainsci101 00664

53. Versino M, Mandala M, Colnaghi S, Ricci G, Faralli M, Ramat S. The integration of multisensory motion stimuli is impaired in vestibular migraine patients. J Neurol. (2020) 267:2842-50. doi: 10.1007/s00415-020-09905-1

54. Casani AP, Lazzerini F, Marconi O, Vernassa N. The role of the functional head impulse test with and without optokinetic stimuli in vestibular migraine and acute unilateral vestibulopathy: discovering a dynamic visual dependence. J Clin Med. (2021) 10:3787. doi: 10.3390/jcm10173787

55. Flook M, Frejo L, Gallego-Martinez A, Martin-Sanz E, Rossi-Izquierdo M, Amor-Dorado JC, et al. Differential proinflammatory signature in vestibular migraine and Meniere disease. Front Immunol. (2019) 10:1229. doi: 10.3389/fimmu.2019.01229

56. Zhang Y, Zhang Y, Tian K, Wang Y, Fan X, Pan Q, et al. Calcitonin gene-related peptide facilitates sensitization of the vestibular nucleus in a rat model of chronic migraine. J Headache Pain. (2020) 21:72. doi: 10.1186/s10194-020-01145-y

57. Wang M, Mason BN, Sowers LP, Kuburas A, Rea BJ, Russo AF. Investigating migraine-like behavior using light aversion in mice. J Vis Exp. (2021) 10.3791/62839. doi: 10.3791/62839

58. Shin JH, Kim YK, Kim HJ, Kim JS. Altered brain metabolism in vestibular migraine: comparison of interictal and ictal findings. Cephalalgia. (2014) 34:58-67. doi: 10.1177/0333102413498940 
59. Obermann M, Wurthmann S, Steinberg BS, Theysohn N, Diener HC, Naegel S. Central vestibular system modulation in vestibular migraine. Cephalalgia. (2014) 34:1053-61. doi: 10.1177/0333102414527650

60. Bednarczuk NF, Bonsu A, Ortega MC, Fluri AS, Chan J, Rust H, et al. Abnormal visuo-vestibular interactions in vestibular migraine: a cross sectional study. Brain. (2019) 142:606-16. doi: 10.1093/brain/awy355

61. Nigmatullina Y, Hellyer PJ, Nachev P, Sharp DJ, Seemungal BM. The neuroanatomical correlates of training-related perceptuo-reflex uncoupling in dancers. Cereb Cortex. (2015) 25:554-62. doi: 10.1093/cercor/bht266

62. Soto E, Vega R, Sesena E. Neuropharmacological basis of vestibular system disorder treatment. J Vestib Res. (2013) 23:119-37. doi: 10.3233/VES-130494

63. King S, Wang J, Priesol AJ, Lewis RF. Central integration of canal and otolith signals is abnormal in vestibular migraine. Front Neurol. (2014) 5:233. doi: 10.3389/fneur.2014.00233

64. King S, Priesol AJ, Davidi SE, Merfeld DM, Ehtemam F, Lewis RF. Self-motion perception is sensitized in vestibular migraine: pathophysiologic and clinical implications. Sci Rep. (2019) 9:14323. doi: 10.1038/s41598-019-50803-y

65. Vass Z, Steyger PS, Hordichok AJ, Trune DR, Jancso G, Nuttall AL. Capsaicin stimulation of the cochlea and electric stimulation of the trigeminal ganglion mediate vascular permeability in cochlear and vertebro-basilar arteries: a potential cause of inner ear dysfunction in headache. Neuroscience. (2001) 103:189-201. doi: 10.1016/S0306-4522(00)00521-2

66. Baloh RW. Vestibular migraine I: mechanisms, diagnosis, and clinical features. Semin Neurol. (2020) 40:76-82. doi: 10.1055/s-0039-3402735

67. Cass SP, Furman JM, Ankerstjerne K, Balaban C, Yetiser S, Aydogan B. Migraine-related vestibulopathy. Ann Otol Rhinol Laryngol. (1997) 106:182-9. doi: 10.1177/000348949710600302

68. Carvalho GF, Vianna-Bell FH, Florencio LL, Pinheiro CF, Dach F, Bigal ME, et al. Presence of vestibular symptoms and related disability in migraine with and without aura and chronic migraine. Cephalalgia. (2019) 39:29-37. doi: 10.1177/0333102418769948

69. Calhoun AH, Ford S, Pruitt AP, Fisher KG. The point prevalence of dizziness or vertigo in migraine-and factors that influence presentation. Headache. (2011) 51:1388-92. doi: 10.1111/j.1526-4610.2011.01970.x

70. Balthazart J, Ball GF. Is brain estradiol a hormone or a neurotransmitter? Trends Neurosci. (2006) 29:241-9. doi: 10.1016/j.tins.2006.03.004

71. Marin R, Diaz M. Estrogen interactions with lipid rafts related to neuroprotection. impact of brain ageing and menopause. Front Neurosci. (2018) 12:128. doi: 10.3389/fnins.2018.00128

72. Park JH, Viirre E. Vestibular migraine may be an important cause of dizziness/vertigo in perimenopausal period. Med Hypotheses. (2010) 75:40914. doi: 10.1016/j.mehy.2009.04.054

73. Baron EP, Cherian N, Tepper SJ. Role of greater occipital nerve blocks and trigger point injections for patients with dizziness and headache. Neurologist. (2011) 17:312-7. doi: 10.1097/NRL.0b013e318234e966

74. Lempert T, von Brevern M. Vestibular migraine. Neurol Clin. (2019) 37:695706. doi: 10.1016/j.ncl.2019.06.003

75. Soto E, Vega R. Neuropharmacology of vestibular system disorders. Curr Neuropharmacol. (2010) 8:26-40. doi: 10.2174/157015910790909511

76. Lapira A. Vestibular migraine treatment and prevention. HNO. (2019) 67:425-8. doi: 10.1007/s00106-019-0661-3

77. Lauritsen CG, Marmura MJ. Current treatment options: vestibular migraine. Curr Treat Options Neurol. (2017) 19:38. doi: 10.1007/s11940-017-0476-Z

78. Byun YJ, Levy DA, Nguyen SA, Brennan E, Rizk HG. Treatment of vestibular migraine: a systematic review and meta-analysis. Laryngoscope. (2021) 131:186-94. doi: 10.1002/lary.28546

79. Obermann M, Strupp M. Current treatment options in vestibular migraine. Front Neurol. (2014) 5:257. doi: 10.3389/fneur.2014.00257

80. Salviz M, Yuce T, Acar H, Karatas A, Acikalin RM. Propranolol and venlafaxine for vestibular migraine prophylaxis: a randomized controlled trial. Laryngoscope. (2016) 126:169-74. doi: 10.1002/lary.25445

81. Beh SC. Vestibular migraine: how to sort it out and what to do about it. J Neuroophthalmol. (2019) 39:208-19. doi: 10.1097/WNO.00000000000 00791

82. Bisdorff AR. Management of vestibular migraine. Ther Adv Neurol Disord. (2011) 4:183-91. doi: 10.1177/1756285611401647
83. Wang F, Wang J, Cao Y, Xu Z. Serotonin-norepinephrine reuptake inhibitors for the prevention of migraine and vestibular migraine: a systematic review and meta-analysis. Reg Anesth Pain Med. (2020) 45:323-30. doi: 10.1136/rapm-2019-101207

84. Ahn SK, Khalmuratova R, Jeon SY, Kim JP, Park JJ, Hur DG, et al. Colocalization of $5-\mathrm{HT} 1 \mathrm{~F}$ receptor and calcitonin gene-related peptide in rat vestibular nuclei. Neurosci Lett. (2009) 465:151-6. doi: 10.1016/j.neulet.2009.09.008

85. Mecklenburg J, Raffaelli B, Neeb L, Sanchez Del Rio M, Reuter U. The potential of lasmiditan in migraine. Ther Adv Neurol Disord. (2020) 13:1756286420967847. doi: 10.1177/1756286420967847

86. Alghadir AH, Anwer S. Effects of vestibular rehabilitation in the management of a vestibular migraine: a review. Front Neurol. (2018) 9:440. doi: 10.3389/fneur.2018.00440

87. Chari DA, Liu YH, Chung JJ, Rauch SD. Subjective cognitive symptoms and Dizziness Handicap Inventory (DHI) performance in patients with vestibular migraine and Meniere's disease. Otol Neurotol. (2021) 42:883-9. doi: 10.1097/MAO.0000000000003081

88. Diener HC, Tassorelli C, Dodick DW, Silberstein SD, Lipton RB, Ashina M, et al. Guidelines of the international headache society for controlled trials of acute treatment of migraine attacks in adults: fourth edition. Cephalalgia. (2019) 39:687-710. doi: 10.1177/0333102419828967

89. van der Zaag-Loonen HJ, van Leeuwen RB. Dizziness causes absence from work. Acta Neurol Belg. (2015) 115:345-9. doi: 10.1007/s13760-014-0404-x

90. Burch RC, Buse DC, Lipton RB. Migraine: epidemiology, burden, and comorbidity. Neurol Clin. (2019) 37:631-49. doi: 10.1016/j.ncl.2019.06.001

91. Rizk H, Agrawal Y, Barthel S, Bennett ML, Doherty JK, Gerend P, et al. Quality improvement in neurology: neurotology quality measurement set. Otolaryngol Head Neck Surg. (2018) 159:603-7. doi: 10.1177/0194599818790947

92. Rizk HG, Sharon JD, Lee JA, Thomas C, Nguyen SA, Meyer TA. Crosssectional analysis of cognitive dysfunction in patients with vestibular disorders. Ear Hear. (2020) 41:1020-7. doi: 10.1097/AUD.0000000000000825

93. Liu YF, Locklear TD, Sharon JD, Lacroix E, Nguyen SA, Rizk HG. Quantification of cognitive dysfunction in dizzy patients using the neuropsychological vertigo inventory. Otol Neurotol. (2019) 40:e723-31. doi: 10.1097/MAO.0000000000002311

94. Sharon JD, Krauter R, Kirk L, Pasquesi L, Allen IE, Formeister EJ, et al. Development and validation of VM-PATHI: vestibular migraine patient assessment tool and handicap inventory. Otol Neurotol. (2020) 41:e494-500. doi: 10.1097/MAO.0000000000002561

Conflict of Interest: NS serves on the speaker's bureau for Abbvie and Amgen as well as an educational consultant to Amgen. FG serves on the speakers bureau for BioHaven and Amgen and as a consultant for Allergan. HD has a patent pending: "Combination therapy for the treatment of vertigo."

The remaining authors declare that the research was conducted in the absence of any commercial or financial relationships that could be construed as a potential conflict of interest.

Publisher's Note: All claims expressed in this article are solely those of the authors and do not necessarily represent those of their affiliated organizations, or those of the publisher, the editors and the reviewers. Any product that may be evaluated in this article, or claim that may be made by its manufacturer, is not guaranteed or endorsed by the publisher.

Copyright (C) 2022 Mallampalli, Rizk, Kheradmand, Beh, Abouzari, Bassett, Buskirk, Ceriani, Crowson, Djalilian, Goebel, Kuhn, Luebke, Mandalà, Nowaczewska, Spare, Teggi, Versino, Yuan, Zaleski-King, Teixido and Godley. This is an open-access article distributed under the terms of the Creative Commons Attribution License (CC $B Y)$. The use, distribution or reproduction in other forums is permitted, provided the original author(s) and the copyright owner(s) are credited and that the original publication in this journal is cited, in accordance with accepted academic practice. No use, distribution or reproduction is permitted which does not comply with these terms. 